\title{
A short note on analysis and application of a stochastic open-ended logistic growth model
}

\author{
Hidekazu Yoshioka (D),e , Yuta Yaegashi ${ }^{\mathrm{b}, \mathrm{c}}$, Yumi Yoshioka ${ }^{\mathrm{a}}$ and Kentaro Tsugihashi ${ }^{\mathrm{d}}$ \\ ${ }^{a}$ Graduate School of Natural Science and Technology, Shimane University, Matsue, Japan; ${ }^{b}$ Graduate School \\ of Agriculture, Kyoto University, Kyoto, Japan; ${ }^{C}$ Japan Society for the Promotion Science, Tokyo, Japan; \\ ${ }^{\mathrm{d}}$ Graduate School of Life and Environmental Science, Shimane University, Matsue, Japan; ${ }^{\mathrm{e}}$ Fisheries \\ Ecosystem Project Center, Shimane University, Matsue, Japan
}

\begin{abstract}
A minimal stochastic generalization of a deterministic open-ended logistic growth model is proposed for efficiently describing the biological growth of individual organisms under natural environment. The model is a system of stochastic differential equations. Its unique solvability in a strong sense is proven, and the behaviour of the solution is analysed. The presented model is then applied to the migratory fish Plecoglossus altivelis altivelis ( $P$. altivelis, Ayu) having a one-year life history based on the data sets collected in 2017 and 2018.
\end{abstract}

\section{ARTICLE HISTORY}

Received 18 January 2019 Accepted 6 November 2019

\section{KEYWORDS}

Open-ended logistic growth; stochastic differential equation; Plecoglossus altivelis altivelis; unique solvability

\section{Introduction}

Evaluation and prediction of biological growth (length, mass or size etc.) of individual organisms are indispensable in resources and ecological management problems, such as agriculture (Dasgupta, 2017; García-Barrios, Mayer-Foulkes, Franco, Urquijo-Vásquez, \& Franco-Pérez, 2001), forestry (Aubry-Kientz, Rossi, Boreux, \& Hérault, 2015; Garcia, 1983) and fishery (Basilone et al., 2018; Yoshioka \& Yaegashi, 2018a). Biological growth in a natural environment can be reasonably considered in the framework of stochastic processes (Wang \& Thomas, 1995), in which each sample path corresponds to each individual, and difference among individuals is naturally described. Mathematical models based on stochastic differential equations (SDEs) (Øksendal, 2003) are major examples, and models based on continuous (Barrera, Román-Román, \& Torres-Ruiz, 2018) and jump (Russo et al., 2009) stochastic processes have been proposed so far. Probabilistic linkages between deterministic and stochastic models have been analysed as well (Di Crescenzo \& Spina, 2016).

Models of the logistic or Ricker types are in the major stream of biological growth models (Tsoularis \& Wallace, 2002). The open-ended model (Thornley \& France, 2005) is a unique deterministic logistic model where the weight and the capacity (final mass) follow a system of differential equations. The model can efficiently describe the biological growth

CONTACT Hidekazu Yoshioka $\otimes$ yoshih@life.shimane-u.ac.jp $\Theta$ Graduate School of Natural Science and Technology, Shimane University, Matsue, Japan; Fisheries Ecosystem Project Center, Shimane University, Matsue, Japan 
depending on environmental conditions such as temperature and nutrition. Detailed theoretical analyses of the open-ended and related logistic models have been carried out from the viewpoint of dynamical systems (Safuan, Towers, Jovanoski, \& Sidhu, 2012; Thornley, Shepherd, \& France, 2007). The open-ended model, owing to its formulation considering the environmental variability, can potentially describe the biological growth of individual organisms under the natural environment; however, the same initial condition always gives the same growth curve because the model is deterministic. A natural question is whether we can formulate its reasonable stochastic counterpart or not. This question is the motivation of this article.

The objective of this article is to formulate a minimal stochastic generalization of the open-ended logistic model such that individual difference observed in biological growth under natural environment is reasonably described. The model is a system of SDEs driven by a continuous Gaussian white noise; the simplest continuous-time noise. A noise term has been added to the governing SDE of the capacity, representing stochastic environmental variability in a simple manner. Well-posedness of the model from the viewpoint of unique solvability in the strong path-wise sense (Capasso \& Bakstein, 2005), which is a theoretically important and non-trivial issue, is discussed and its solution behaviour is analysed. In addition, as a prompt report, its application to biological growth analysis of the migratory fish Plecoglossus altivelis altivelis ( $P$. altivelis, Ayu) in Hii River, Japan (Yoshioka \& Yaegashi, 2018b) in 2017 and 2018, is carried out. This article thus contributes to the formulation of a stochastic open-ended logistic model, its theoretical analysis and further its application.

\section{Mathematical model}

\subsection{Model formulation}

We consider the biological growth of individual organisms in a habitat with an emphasis on applications to fisheries where tracking growth dynamics of fishes are of critical importance. It has been found, as discussed in Section 3 with the observed data, that their growth dynamics seem to be logistic-like but not homogenous. This means a natural fact that different individuals have different growth curves. We consider that there exists a governing system of SDEs to describe the biological growth dynamics and that the difference among individuals is a consequence of different paths of the noises. SDEs are fundamental tools for modelling and analysis of biological growth dynamics (Donnet, Foulley, \& Samson, 2010; Román-Román \& Torres-Ruiz, 2015; Strathe, Sørensen, \& Danfaer, 2009). They provide a robust framework for assessing the dynamics in a relatively simple manner. In addition, SDEs can be effectively incorporated into modern fisheries management models (Poudel \& Sandal, 2015; Yoshioka, Yaegashi, Yoshioka, \& Tsugihashi, 2019). The stochasticity in our application can be considered as environmental and hydrological disturbance acting on the individual fishes in rivers. Based on the open-ended logistic formalism (Thornley \& France, 2005), the disturbance is efficiently considered to be a factor that distinguishes the growth dynamics of the individuals. The difference between the conventional open-ended logistic model and our model is that the latter has a stochastic term, while the former does not. The disturbance in our model is considered as a continuous-time white noise in the governing SDE of the final mass. Mathematically, we consider that different sample paths 
generated by the system of SDEs correspond to different growth curves. Later, we numerically show that the individuals have different final masses and that the presented model can reasonably reproduce the observed growth dynamics of fishes living in a river.

Mathematical background of SDEs are found in the widely read textbooks (Capasso \& Bakstein, 2005; Øksendal, 2003). The time is denoted as $t \geq 0$ and the body weight at the time $t$ is denoted as a continuous-time variable $X_{t}$. The 1-D standard Brownian motion defined on the usual complete probability space (Øksendal, 2003) is denoted as $B_{t}$. The Brownian motion is a zero-mean Gaussian white noise having independent increments. The variance of $B_{t}-B_{s}(t \geq s \geq 0)$ is $t-s$. The (environmental) capacity, which is referred to as the final mass parameter in Thornley and France (2005), at the time $t$ is denoted as a continuous-time variable $K_{t}$. Based on the deterministic open-ended model (Thornley \& France, 2005), the stochastic counterpart is proposed as

$$
\mathrm{d} X_{t}=r\left(1-\frac{X_{t}}{K_{t}}\right) X_{t} \mathrm{~d} t, \quad t>0
$$

with

$$
\mathrm{d} K_{t}=D\left(X_{t}-K_{t}\right) \mathrm{d} t+\sigma\left(X_{t}-K_{t}\right) \mathrm{d} B_{t}, \quad t>0
$$

subject to the initial condition $0<X_{0}<K_{0}$. Here, $r>0$ represents the specific growth rate, $D>0$ is a relaxation parameter and $\sigma \geq 0$ represents the environmental noise intensity. Setting $\sigma=0$ in (2) recovers the deterministic model (Thornley \& France, 2005). The first SDE (1) is the logistic-type equation having the final mass that is state-dependent in our model. The second SDE (2) governs the temporal evolution of the capacity. Its righthand side has two terms: the first term represents the empirical process of getting older and development in a lumped manner, and the second term its fluctuation. Phenomenologically, larger $D$ results in an earlier termination of the effective biological growth because the first term has a mean-reverting form like the Ornstein-Uhlenbeck model (Uhlenbeck \& Ornstein, 1930). The model assumes that no body growth occurs when $X_{t}=K_{t}$ as in the classical logistic models. Anderson, Jovanoski, Sidhu, and Towers (2016) also considered a stochastic capacity, but their approach is different from ours because their final mass is essentially a Brownian motion.

\subsection{Theoretical analysis}

Well-posedness, namely unique solvability, of the system (1)-(2) is a non-trivial issue since its coefficients do not comply with the Lipschitz continuity (a sufficient condition for unique solvability of SDEs) (Theorem 4.4; Capasso \& Bakstein, 2005). We prove that the system (1)-(2) admits a unique strong solution. Analogous mathematical results would hold true when $X_{t} / K_{t}$ in (1) is replaced by $\left(X_{t} / K_{t}\right)^{\theta}$, where $\theta \geq 1$ as in Thornley et al. (2007).

Firstly, observe that a formal application of the Itô's lemma (Øksendal, 2003) to $U_{t}=X_{t} / K_{t}$ yields

$$
\begin{aligned}
\mathrm{d} U_{t} & =\frac{\mathrm{d} X_{t}}{K_{t}}-\frac{X_{t} \mathrm{~d} K_{t}}{K_{t}^{2}}+\frac{1}{2} \frac{2 X_{t}\left(\mathrm{~d} K_{t}\right)^{2}}{K_{t}^{3}} \\
& =A\left(U_{t}\right) \mathrm{d} t+C\left(U_{t}\right) \mathrm{d} B_{t}
\end{aligned}
$$


with

$$
A\left(U_{t}\right)=U_{t}\left(1-U_{t}\right)\left(r+D+\sigma^{2}\left(1-U_{t}\right)\right)
$$

and

$$
C\left(U_{t}\right)=\sigma U_{t}\left(1-U_{t}\right)
$$

The following lemma is the key to show the well-posedness of the model.

Lemma 2.1: The SDE

$$
d U_{t}=A\left(U_{t}\right) d t+C\left(U_{t}\right) d B_{t}, t>0
$$

with the initial condition $0<U_{0}<1$ admits a unique strong solution with $0<U_{t}<1$.

Proof: The proof here follows Appendix A of Lungu and Øksendal (1997). The only difference is that our SDE (6) has a cubic drift term, while that of the literature has a quadratic drift term. Firstly, consider the auxiliary SDE

$$
\mathrm{d} U_{t}=\bar{A}\left(U_{t}\right) \mathrm{d} t+\max \left\{0, C\left(U_{t}\right)\right\} \mathrm{d} B_{t}, \quad t>0
$$

with

$$
\bar{A}\left(U_{t}\right)=\left\{\begin{array}{cc}
A\left(U_{t}\right) & \left(0 \leq U_{t} \leq 1\right) \\
0 & \left(U_{t}<0, U_{t}>1\right)
\end{array}, \quad t>0\right.
$$

which has globally Lipschitz continuous drift and diffusion terms, and thus admits a unique strong solution (Theorem 4.4 of Capasso \& Bakstein, 2005). Therefore, if the solution to the auxiliary SDE $(7)$ is valued in $(0,1)$, then it is a unique strong solution to the SDE (6) as well.

Set the stopping time $\tau=\inf \left\{t>0 \mid U_{t}=0,1\right\}$ and consider the process $Y_{t}=\ln U_{t}$ defined in $0 \leq t<\tau$. We show $\tau=+\infty$, meaning that $U_{t}>0$ for $t \geq 0$. Assume $U_{\tau}=0$. An application of the Itô's lemma to $Y_{t}$ yields

$$
\begin{aligned}
\mathrm{d} Y_{t} & =\frac{\mathrm{d} U_{t}}{U_{t}}-\frac{\left(\mathrm{d} U_{t}\right)^{2}}{2 U_{t}^{2}} \\
& =\left(1-U_{t}\right)\left(r+D+\sigma^{2}\left(1-U_{t}\right)\right) \mathrm{d} t+\sigma\left(1-U_{t}\right) \mathrm{d} B_{t}-\frac{1}{2} \sigma^{2}\left(1-U_{t}\right)^{2} \mathrm{~d} t
\end{aligned}
$$

and thus

$$
Y_{t}=Y_{0}+\int_{0}^{t}\left[\left(1-U_{s}\right)\left(r+D+\sigma^{2}\left(1-U_{s}\right)\right)-\frac{1}{2} \sigma^{2}\left(1-U_{s}\right)^{2}\right] \mathrm{d} s+\int_{0}^{t} \sigma\left(1-U_{s}\right) \mathrm{d} B_{s} .
$$

The right-hand side of (10) does not blow up at each $t \geq 0$, while the left hand-side blows up as $t \uparrow \tau$ by the definition of $\tau$. This is a contradiction, showing $\tau=+\infty$. In the same way, $U_{t}<1$ for $t \geq 0$ follows by considering the process $Y_{t}=\ln \left(1-U_{t}\right)$. Consequently, we proved that the solution to the $\operatorname{SDE}(7)$ is valued in $(0,1)$. This completes the proof. 
Consider the system of SDEs

$$
\mathrm{d} X_{t}=r\left(1-U_{t}\right) X_{t} \mathrm{~d} t, t>0
$$

and (6) with the initial conditions $X_{0}>0$ and $0<U_{0}<1$. By Lemma 2.1, $U_{t}$ is bounded and continuous, showing that the SDE (11) is uniquely solved as

$$
X_{t}=X_{0} \exp \left(\int_{0}^{t} r\left(1-U_{s}\right) \mathrm{d} s\right)>0, \quad t \geq 0 .
$$

Therefore, the systems (11) and (6) admits a unique strong solution $\left(X_{t}, U_{t}\right), t \geq 0$. We then apply the Itô's lemma to $K_{t}=X_{t} / U_{t}$ and yield the SDE (2). Furthermore, we have $0<X_{t}<K_{t}$ because $0<U_{t}<1$ and $X_{t}>0$. The presented model thus generates exactly one sample path such that $0<X_{t}<K_{t}$ is satisfied for each given $B_{t}$. Since

$$
U_{t}\left(1-U_{t}\right)(r+D) \leq A\left(U_{t}\right) \leq U_{t}\left(1-U_{t}\right)\left(r+D+\sigma^{2}\right)
$$

for the solution $U_{t}$, the comparison argument of SDEs (Corollary 3.1 of Peng \& Zhu, 2006) with the limiting behaviour of a logistic SDE (Theorem 2.2 of Lungu \& Øksendal, 1997) gives $\lim _{t \rightarrow+\infty} U_{t}=\lim _{t \rightarrow+\infty} X_{t} K_{t}^{-1}=1$. The biological growth, therefore, encounters an eventual saturation as in the conventional models.

Consequently, we arrive at the following main mathematical analysis result.

Theorem 2.1: The system (1)-(2) admits a unique strong solution with $0<X_{t}<K_{t}$ for $t \geq 0$. This $X_{t}$ is increasing with respect to $t>0$. In addition, $\lim _{t \rightarrow+\infty} X_{t} K_{t}^{-1}=1$.

However, these results do not give quantitative information on the biological growth. This issue is addressed below through an application.

Note that our analysis results have not only mathematical meaning but also biological implications. From a biological viewpoint, Theorem 2.1 shows that the meaning of the variables is in accordance with our intuitions because $0<X_{t}<K_{t}$ is satisfied. In addition, we see that the presented model leads to non-decreasing sample paths of $X_{t}$. This implies that the body weight of each individual does not decrease with respect to the time $t$, which can be a reasonable requirement in mathematical modelling of biological growth curves (Filipe, Braumann, \& Roquete, 2012; Román-Román \& Torres-Ruiz, 2015). On the other hand, the final mass $K_{t}$ is not necessarily monotone as numerically demonstrated in the next section. From a mathematical viewpoint, the authors consider that still many biological models have not been analysed mathematically, meaning that their well-posedness is an open question. Using a model that is not necessarily well-posed is dangerous and potentially leads to wrong results. Theorem 2.1 guarantees the well-posedness of our model. In summary, the model is biologically plausible and mathematically rigorous.

Remark 2.1: We can consider a stochastic term in the SDE of $X_{t}$ as well. Mathematically, this seems not to be a difficult task and the unique solvability of the resulting system of SDEs would still hold true. In addition, a more complex model may be more accurate. However, the increasing property of the body weight $X_{t}$ proved in Theorem 2.1 will be lost by adding the noise term. Therefore, the resulting model may be qualitatively different from the presented 
model. Furthermore, considering more than one noise sources requires modelling their correlations. If a sufficiently dense data set will be available, then we will be able to address this issue.

\section{Application}

\subsection{Parameter identification procedure}

The presented model is applied to P. altivelis in Hii River, San-in area, Japan. Detailed life history of the fish is explained in Yoshioka and Yoshioka and Yaegashi (2018a, 2018b, 2018c). In this river, most of the fish population originate from juvenile fish released in early May by Hii River Fisheries Cooperatives. The main growth period of the fish in the river is from May to October each year, after that they spawn and die.

The two field surveys were carried out during 2017 and 2018 in the river with the help of Hii River Fisheries Cooperatives. The first one is intensive surveys on 3 August 2017 and 6 August 2018, at which 234 and 189 individuals were caught, respectively. The second one is the long-term field survey from July to October each year, in which the fish is caught in the river at irregular intervals. In both surveys, the body weights of the caught individual fishes were measured. A part of the data collected at the intensive and field surveys in 2017 has been presented in Yoshioka et al. (2019) and the annual information booklet of Hii River Fisheries Cooperatives (Yoshioka, Yaegashi, Yoshioka, \& Tsugihashi, 2018).

For each year, the model parameters are identified with the collected data at the intensive surveys with a trial and error approach, so that the statistical moments (mean, standard deviation and skewness) and the histogram are reasonably reproduced. The statistical moments of $X_{t}$ are computed with a Monte-Carlo method with the standard Euler-Maruyama discretization (Kloeden, Platen, \& Schurz, 1994). The total number of generated paths are $10^{5}$ and the time increment is 90/45,000 (day). At each time step, we simply computed the sample mean, sample standard deviation and sample skewness using the sample paths generated by the Monte-Carlo method. The initial time is set so that the day of intensive survey is 90 (day) and the initial condition $X_{0}$ is set as $6(\mathrm{~g})$ based on a preliminary observation result. The parameters to be identified are thus $K_{0}(\mathrm{~g}), r(1 /$ day), $D$ $\left(1 /\right.$ day) and $\sigma\left(1 /\right.$ day $\left.^{1 / 2}\right)$. For each year, the identified model is validated with the long-term field survey results. We assume the same value of $K_{0}$ for both 2017 and 2018 considering that the difference in biological conditions of the juveniles between each year was small.

\subsection{Application results}

Table 1 summarizes the identified parameter values and Table 2 compares the observed statistical moments and those with the identified model parameters. Table 2 implies that models that do not consider the individual difference, namely deterministic models, are clearly not applicable in the present case, and that the observed distributions are positively skewed. This is because deterministic models are in principle not able to capture probabilistic distributions. The difference between the observed data and the presented model in Table 2 can be due to model simplicity; the presented model is a minimal open-ended stochastic logistic model.

Figure 1 plots sample paths with the data in 2018, supporting Theorem 2.1 numerically. In addition, Figure 2 implies that different sample paths have different final masses. 
Table 1. Summary of identified model parameters.

\begin{tabular}{llr}
\hline & 2017 & 2018 \\
\hline$K_{0}(\mathrm{~g})$ & \multicolumn{2}{c}{200} \\
$r(1 /$ day $)$ & 0.038 & 0.042 \\
$D(1 /$ day $)$ & 0.015 & 0.019 \\
$\sigma\left(1 /\right.$ day $\left.^{1 / 2}\right)$ & 0.11 & 0.091 \\
\hline
\end{tabular}

Table 2. Comparison of observed and modelled statistical moments.

\begin{tabular}{lccccc}
\hline & \multicolumn{2}{c}{2017} & & \multicolumn{2}{c}{2018} \\
\cline { 2 - 3 } \cline { 6 - 7 } & Observation & Model & & Observation & Model \\
\hline Mean (g) & 55.6 & 55.5 & & 57.3 & 57.3 \\
Standard deviation (g) & 19.1 & 19.2 & & 18.5 & 18.9 \\
Skewness (-) & 0.78 & 0.91 & & 1.16 & 1.14 \\
\hline
\end{tabular}

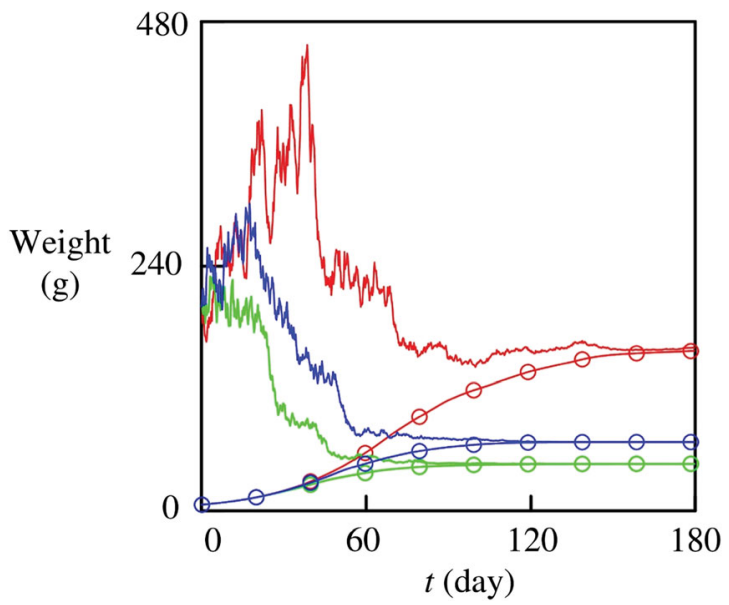

Figure 1. Sample paths of the couple $\left(X_{t}, K_{t}\right)$ with the data in 2018. The same colour indicates the variables in the same couple. Paths with $\bigcirc$ correspond to $X_{t}$, and those without $\bigcirc$ to $K_{t}$. Different colors correspond to different sample paths. For the colored figure, see the online version.

Figures 2 and 3 compare the modelled and observed histograms at the intensive surveys in 2017 and 2018. The results obtained in Table 2 demonstrate that the presented model accurately reproduces the observed statistical moments. The histograms with the observed data in Figures 2 and 3 are also well reproduced by the histograms generated by the presented model. In particular, the positively skewed observed distributions are captured by the model. From Table 1, we see that the biological growth was subject to smaller growth rate, smaller relaxation rate and larger noise intensity in 2017 than those in 2018.

Finally, our model is examined against the collected data by the long-term surveys in 2017 and 2018. Figures 4 and 5 compare the modelled (mean, mean \pm standard deviation and skewness) and observed biological growth of the individuals from May $1(t=0)$ in each year. The figures demonstrate that the model reasonably tracks the observation results despite its simplicity. The obtained results show that most of the observed data plots are within the range of the mean at most the standard deviation. In each figure, the mean 


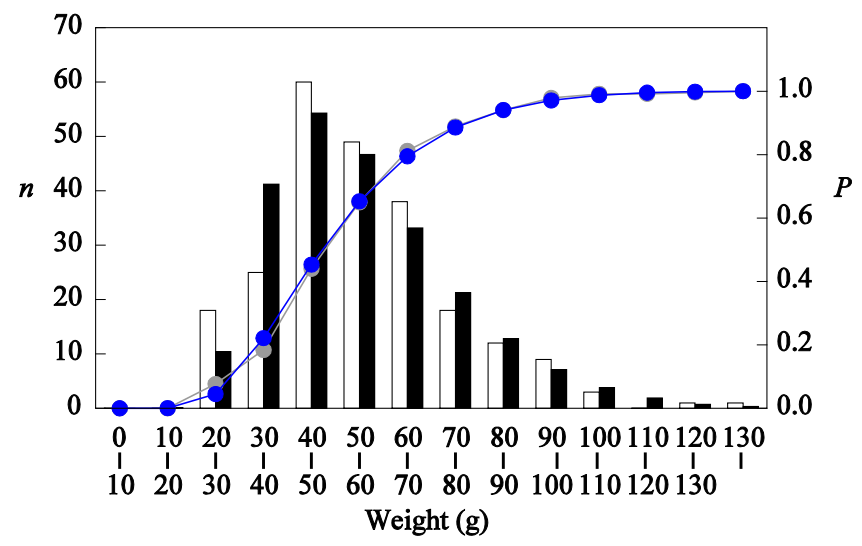

Figure 2. Comparison of the modelled and observed data at the intensive survey in 2017. Bars (White: observed, Black, modelled) represent the histograms. Piecewise lines with circles (Grey: observed, Blue: modelled) represent the cumulative probability. ' $0-10^{\prime}$ means ' $0 \leq X<10^{\prime}$. The same holds true for the others. For the colored figure, see the online version.

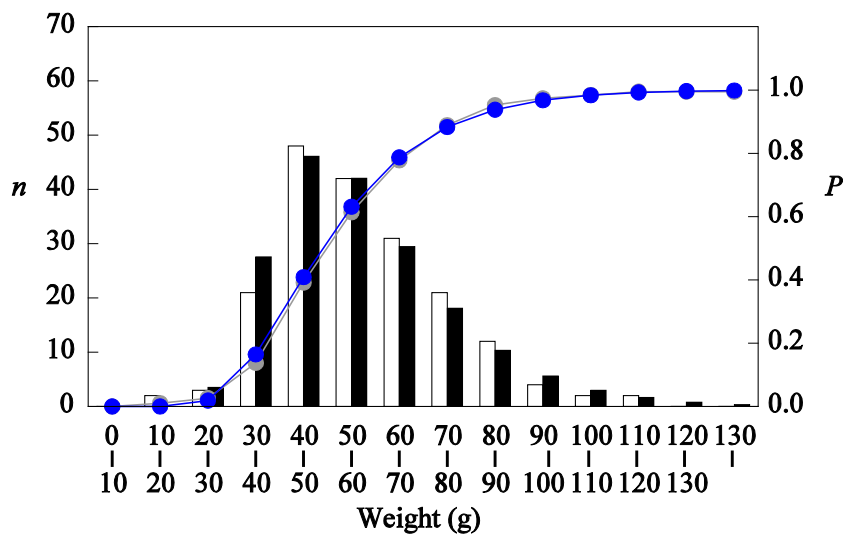

Figure 3. Comparison of the modelled and observed data at the intensive survey in 2018. Comparison of the modelled and observed histograms in 2017. The same legends with Figure 2.

growth curve has a sigmoid-like shape as in the standard deterministic logistic models. Comparing Figure 1, Figures 4 and 5 show that both sample paths and their means have sigmoid-like shapes having different final masses. Unfortunately, because of a technical reason that individuals die after measuring their weights, it was difficult to experimentally verify whether the individuals have sigmoid-like paths as shown in Figure 1. A contactless methodology to correctly track the growth of individual fishes in the natural river environment must be developed to resolve this issue.

The modelled results suggest qualitatively the same growth of the fish between each year, while larger stochasticity in the growth in 2017. In addition, the positive skewness for the latter half of the computational period is consistent with the observation results that the individuals having higher weights than the mean values are captured more frequently. Another important finding is that the growth rate $r$ in 2018 is larger than that in 2017 as shown in Table 1, and the mean body weight is larger in 2018 than that in 2017 at the 


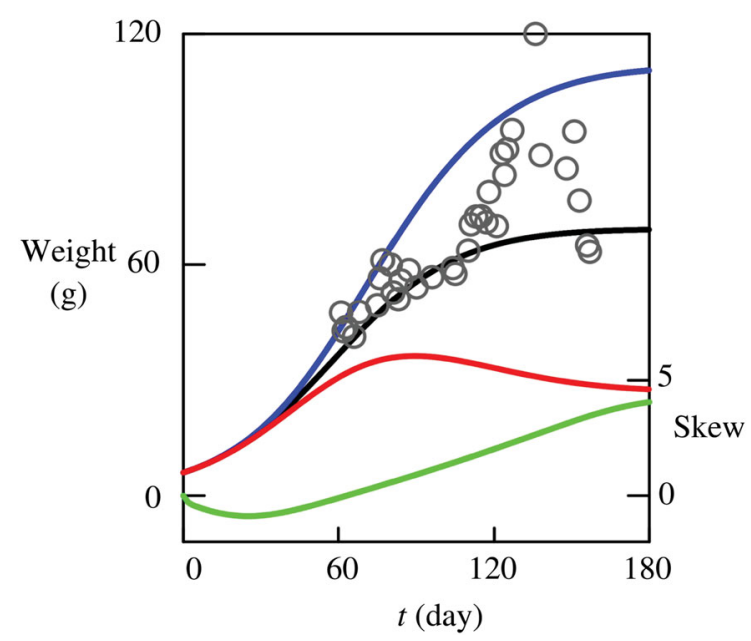

Figure 4. Comparison of the modelled and the long-term observation result in 2017. Circles represent the observed data, and the curves represent the modelled results (Black: mean, Blue: mean + standard deviation, Red: mean - standard deviation, Green: skewness). For the colored figure, see the online version.

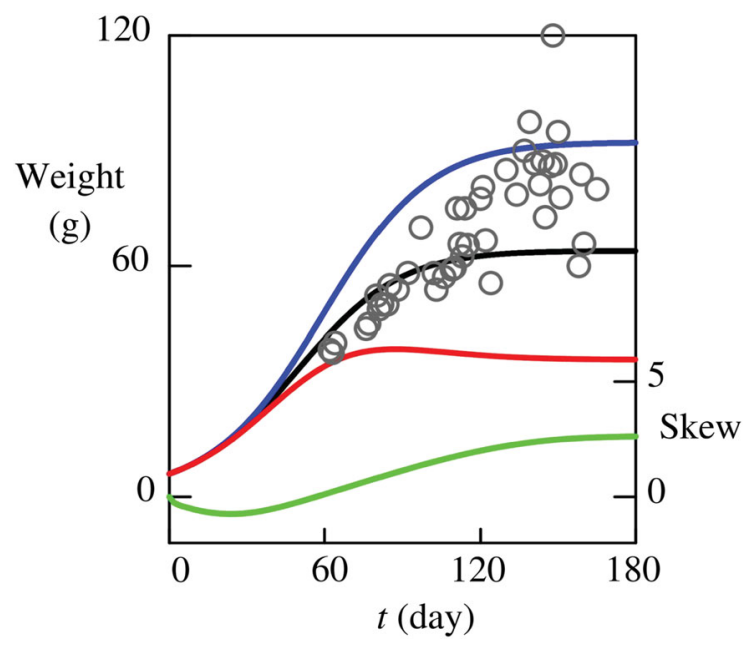

Figure 5. Comparison of the modelled and the long-term observation result in 2018 . The same legends with Figure 4.

intensive surveys in August as shown in Table 2. August is the peak season of harvesting the fish in Hii River, implying that a greater number of larger fishes are captured in 2018 than in 2017. The mean body weight at 180 (day) around which the fish reproduces is 63.9 (g) in 2018 and 69.1 (g) in 2017. The opposite ordering of the means between the results at the intensive surveys and those at the terminal time may be due to the larger relaxation parameter $D$ (earlier maturity) in 2018. An allometric scaling relationship (Hendriks \& Mulder, 2008), which considers the fecundity as an increasing function of the body weight, implies that a larger number of eggs have been reproduced per individual in 2017. 


\section{Conclusions}

A stochastic generalization of the open-ended logistic model was presented and its mathematical analysis was carried. The model was well-posed and its parameter values were successfully identified with the collected data. The biological growth with the model is monotone in the mean and encounters an eventual saturation like the conventional logistic models. Future research will focus on an application of the model to fisheries management, where the central problem would be cost-effective as well as ecologically-conscious fisheries planning. Applicability of the model to other organisms is also of great interest.

\section{Acknowledgements}

The authors thank Hii River Fishery Cooperatives for their kind help in our field surveys. The critical comments from the anonymous reviewers considerably improved the contents of the manuscript.

\section{Disclosure statement}

No potential conflict of interest was reported by the authors.

\section{Funding}

Japan Society for the Promotion of Science (JSPS) research grant 17K15345 and a grant for the ecological survey of a life history of the landlocked ayu Plecoglossus altivelis altivelis from MLIT support this research.

\section{ORCID}

Hidekazu Yoshioka (D) http://orcid.org/0000-0002-5293-3246

\section{References}

Anderson, C., Jovanoski, Z., Sidhu, H. S., \& Towers, I. N. (2014). Logistic equation is a simple stochastic carrying capacity. ANZIAM Journal, 56, 431-445.

Aubry-Kientz, M., Rossi, V., Boreux, J. J., \& Hérault, B. (2015). A joint individual-based model coupling growth and mortality reveals that tree vigor is a key component of tropical forest dynamics. Ecology and Evolution, 5, 2457-2465.

Barrera, A., Román-Román, P., \& Torres-Ruiz, F. (2018). A hyperbolastic type-I diffusion process: Parameter estimation by means of the firefly algorithm. Biosystems, 163, 11-22.

Basilone, G., Ferreri, R., Mangano, S., Pulizzi, M., Gargano, A., Barra, M., .. Bonanno, A. (2018). Effects of habitat conditions at hatching time on growth history of offspring European anchovy, Engraulis encrasicolus, in the Central Mediterranean Sea. Hydrobiologia, 821, 99-111.

Capasso, V., \& Bakstein, D. (2005). An introduction to continuous-time stochastic processes. Boston: Birkhauser.

Dasgupta, R. (2017). Growth of tuber crops and almost sure band for quantiles. Communications in Statistics - Simulation and Computation, 46(2), 1062-1085.

Di Crescenzo, A., \& Spina, S. (2016). Analysis of a growth model inspired by Gompertz and Korf laws, and an analogous birth-death process. Mathematical Biosciences, 282, 121-134.

Donnet, S., Foulley, J. L., \& Samson, A. (2010). Bayesian analysis of growth curves using mixed models defined by stochastic differential equations. Biometrics, 66, 733-741.

Filipe, P. A., Braumann, C. A., \& Roquete, C. J. (2012). Multiphasic individual growth models in random environments. Methodology and Computing in Applied Probability, 14, 49-56.

Garcia, O. (1983). A stochastic differential equation model for the height growth of forest stands. Biometrics, 39(4), 1059-1072. 
García-Barrios, L., Mayer-Foulkes, D., Franco, M., Urquijo-Vásquez, G., \& Franco-Pérez, J. (2001). Development and validation of a spatially explicit individual-based mixed crop growth model. Bulletin of Mathematical Biology, 63(3), 507-526.

Hendriks, A. J., \& Mulder, C. (2008). Scaling of offspring number and mass to plant and animal size: Model and meta-analysis. Oecologia, 155, 705-716.

Kloeden, P. E., Platen, E., \& Schurz, H. (1994). Numerical solution of SDE through computer experiments. Berlin: Springer Science \& Business Media.

Lungu, E. M., \& Øksendal, B. (1997). Optimal harvesting from a population in a stochastic crowded environment. Mathematical Biosciences, 145(1), 47-75.

Øksendal, B. (2003). Stochastic differential equations. Berlin: Springer.

Peng, S., \& Zhu, X. (2006). Necessary and sufficient condition for comparison theorem of 1-dimensional stochastic differential equations. Stochastic Processes and their Applications, 116(3), 370-380.

Poudel, D., \& Sandal, L. K. (2015). Stochastic optimization for multispecies fisheries in the Barents Sea. Natural Resource Modeling, 28, 219-243.

Román-Román, P., \& Torres-Ruiz, F. (2015). A stochastic model related to the Richards-type growth curve. Estimation by means of simulated annealing and variable neighborhood search. Applied Mathematics and Computation, 266, 579-598.

Russo, T., Baldi, P., Parisi, A., Magnifico, G., Mariani, S., \& Cataudella, S. (2009). Lévy processes and stochastic von Bertalanffy models of growth, with application to fish population analysis. Journal of Theoretical Biology, 258(4), 521-529.

Safuan, H. M., Towers, I., Jovanoski, Z., \& Sidhu, H. (2012). Coupled logistic carrying capacity model. ANZIAM Journal, 53, 172-184.

Strathe, A. B., Sørensen, H., \& Danfaer, A. (2009). A new mathematical model for combining growth and energy intake in animals: The case of the growing pig. Journal of Theoretical Biology, 261, $165-175$.

Thornley, J. H., \& France, J. (2005). An open-ended logistic-based growth function. Ecological Modelling, 184(2-4), 257-261.

Thornley, J. H., Shepherd, J. J., \& France, J. (2007). An open-ended logistic-based growth function: Analytical solutions and the power-law logistic model. Ecological Modelling, 204(3-4), 531-534.

Tsoularis, A., \& Wallace, J. (2002). Analysis of logistic growth models. Mathematical Biosciences, 179(1), 21-55.

Uhlenbeck, U. E., \& Ornstein, L. S. (1930). On the theory of the Brownian motion. Physical Review, $36,823-841$.

Wang, Y. G., \& Thomas, M. R. (1995). Accounting for individual variability in the von Bertalanffy growth model. Canadian Journal of Fisheries and Aquatic Sciences, 52(7), 1368-1375.

Yoshioka, H., \& Yaegashi, Y. (2018a). Mathematical analysis for management of released fish. Optimal Control Applications and Methods, 39(2), 1141-1146.

Yoshioka, H., \& Yaegashi, Y. (2018b). Stochastic differential game for management of non-renewable fishery resource under model ambiguity. Journal of Biological Dynamics, 12(1), 817-845.

Yoshioka, H., \& Yaegashi, Y. (2018c). Mathematical analysis for management of released fish. Optimal Control Applications and Methods, 39(2), 1141-1146.

Yoshioka, H., Yaegashi, Y., Yoshioka, Y., \& Tsugihashi, K. (2018). A mathematical challenge to comprehend the current environmental and ecological conditions of Hii River, Seoto (An annual report of inland fisheries in Hii River), Hii River Fisheries Cooperatives, pp. 6-7. (in Japanese). Retrieved from http://hiikawafish.lolipop.jp/hiikawafish/date/180701_seoto_no11.pdf

Yoshioka, H., Yaegashi, Y., Yoshioka, Y., \& Tsugihashi, K. (2019). Optimal harvesting policy of aninland fishery resource under incomplete information. Applied Stochastic Models in Business and Industry, 35(4), 939-962. 